\title{
In situ near-infrared spectroscopic studies of the structural changes of polyethylene during melting
}

\author{
Masami Mizushima, Takanobu Kawamura, Kenji Takahashi and Koh-hei Nitta \\ Conformational changes occurring during the melting of various polyethylene (PE) materials, including high-density PE (HDPE), \\ linear low-density PE (LLDPE) and low-density polyethylene (LDPE), were investigated using near-infrared spectroscopy. The \\ assignment of PE to the 1650-1900 nm spectral region was suggested, on the basis of computational and experimental data for \\ normal alkanes. The present results suggested that the 1690 and $1710 \mathrm{~nm}$ bands should be assigned to the $\mathrm{CH}_{3}$ groups in \\ branched chains and chain ends, respectively. The HDPE and LDPE crystal lattices have very few defects, in contrast to LLDPE, \\ which has more crystal lattice defects. The chain ends and junctions of LDPE are excluded from the crystal lattice and exist in a \\ thick amorphous layer, whereas LLDPE includes comonomers in its crystalline lattice.
}

Polymer Journal (2012) 44, 162-166; doi:10.1038/pj.2011.100; published online 9 November 2011

Keywords: melting process; near-infrared; polyethylene

\section{INTRODUCTION}

The structural rearrangement and phase transformation of semicrystalline polymers, such as polyethylene (PE) and polypropylene, during annealing and heating have received significant academic and industrial interest. The thermal and melting properties of these semicrystalline polymers have been extensively investigated; however, the molecular mechanisms of melting and reorganization are still not well understood. The main reason for this is the paucity of reported in situ measurements of structural morphology and chain conformation during melting and heating processes.

Fourier transform infrared spectroscopy is a powerful tool for detecting chain conformation and structural change or phase transition during heating and cooling. In situ Fourier transform infrared spectroscopic studies of the conformational changes of semicrystalline polymers such as PE and polypropylene have been conducted. ${ }^{1-3}$ The mid-infrared region from $2500-25000 \mathrm{~nm}$ is diagnostic for these polyolefins. However, the very high absorption in the mid-infrared region makes it difficult to assign spectra for industrial polyolefin bulk specimens; very thin films are usually required.

In the near-infrared (NIR) region, we consider absorption and luminescence spectra at wavelengths from $800-2500 \mathrm{~nm}$, in which combination tones and overtones generated by anharmonic molecular vibrations are observed. Their absorption peaks are relatively weak compared with those of the basic tones in the mid-infrared region. These low absorption coefficients enable the nondestructive and direct measurement of vibrational spectra of bulk materials and industrial products. One disadvantage of NIR spectra is the absorption overlap of overtones and combination tones, which complicates the assignment of bands for more complex organic materials.

In such circumstances, Norris et al. ${ }^{4}$ proposed a band assignment method for NIR spectra using statistical techniques. Barton and Noda et al..$^{5-8}$ also assigned NIR bands by correlation with IR bands using two-dimensional correlation spectroscopy. Ozaki et al. and Siesler et al..$^{9-16}$ recently demonstrated that NIR spectroscopy can be useful for the structural characterization of polymeric materials, foods and bio-based materials.

Watanabe et al. ${ }^{17-19}$ demonstrated the applicability of NIR spectroscopy for characterizing thermally induced structural changes in PE. They monitored the temperature-induced phase transition of crystalline PE materials using NIR spectroscopy. They demonstrated how NIR spectroscopy could detect the melting behavior of crystalline phases in various PE materials but did not characterize the conformational changes during heating. Few studies have focused on the conformational changes of crystallizable polymers during the melting process. The aim of this study is to explore the conformational change of typical linear and branched PE materials during the melting process using in situ NIR spectroscopy. NIR spectroscopy makes it possible to analyze the molecular or structural state in actual materials and industrial products.

In this study, we assigned NIR bands to PE chains based on computational and experimental spectra of normal alkanes. The conformational changes of PE chains were investigated under a constant heating rate up to the melting point. 


\section{EXPERIMENTAL PROCEDURE}

\section{Materials}

Commercial-grade samples of high-density polyethylene (HDPE), linear lowdensity polyethylene (LLDPE) with ethyl branches and low-density polyethylene (LDPE) produced by an autoclave reactor were used. The molecular characteristics of these samples are given in Table 1.

PE pellets were melted in a hot press at $483 \mathrm{~K}$ under $10 \mathrm{MPa}$ for $10 \mathrm{~min}$, with subsequent quenching from the melt to $373 \mathrm{~K}$. The thickness of the compressed molded sheets was $\sim 500 \mu \mathrm{m}$

\section{Characterization}

Differential scanning calorimetry (DSC) measurements were carried out using a Diamond DSC (PerkinElmer Inc., Waltham, MA, USA). Samples were heated from $313-473 \mathrm{~K}$ at a scanning rate of $20 \mathrm{~K} \mathrm{~min}^{-1}$ under a $\mathrm{N}_{2}$ atmosphere. The crystallinity in the weight fraction was determined from fusion enthalpy data, where the equilibrium fusion enthalpy of PE was taken to be $277.1 \mathrm{~kJ} \mathrm{~kg}^{-1} .20$ The density of sheet samples was obtained with the Archimedes method using a METTLER electrobalance XS205 (Mettler Toredo International Inc., Greifensee, Switzerland), with ethanol as the solvent. The melting temperatures, crystallinity and density of the polymer samples are given in Table 2 .

\section{NIR spectral measurements during melting}

The experimental setup is illustrated in Figure 1. A Xe short-arc lamp (USHIO Inc., Tokyo Japan, UXL-300D-O), InGaAs PIN photodiode (Hamamatsu photonics Inc., Shizuoka, Japan, G8371-03) and monochromator (Hamamatsu photonics Inc. CT-10) were used as the light source, detector and monochromator, respectively. A digital oscilloscope (Lecroy Co., Chestnut Ridge, NY, USA, Model 9310 L) was used to capture spectral data. Spectral data from the

Table 1 Molecular characteristics of the samples

\begin{tabular}{lcccc}
\hline Sample & $\mathrm{M}_{w} \times 10^{-4}$ & $\mathrm{M}_{n} \times 10^{-4}$ & $\mathrm{M}_{w} \mathrm{M}_{n}$ & SCB/mol\% \\
\hline HDPE & 10 & 1.7 & 5.9 & 0 \\
LLDPE & 11 & 2.6 & 4.2 & 3.6 \\
LDPE & 20 & 2.2 & 9.1 & 1.4 \\
\hline
\end{tabular}

Abbreviation: SCB, short chain branch content.

Table 2 Structural characteristics of the sample specimens

\begin{tabular}{lcccc}
\hline Sample & $T_{m} / K^{\mathrm{a}}$ & $\Delta H / \mathrm{kJ} \mathrm{kg}^{-1_{\mathrm{b}}}$ & $\chi_{w} / \%^{\mathrm{c}}$ & ${\text { Density } / \mathrm{kg} \mathrm{m}^{-3}}^{-3}$ \\
\hline HDPE & 137 & 197 & 71 & 955 \\
LLDPE & 123 & 97 & 35 & 915 \\
LDPE & 108 & 92 & 34 & 909 \\
\hline
\end{tabular}

aMelting temperature obtained by DSC

bFusion enthalpy obtained by DSC.

c Crystallinity.

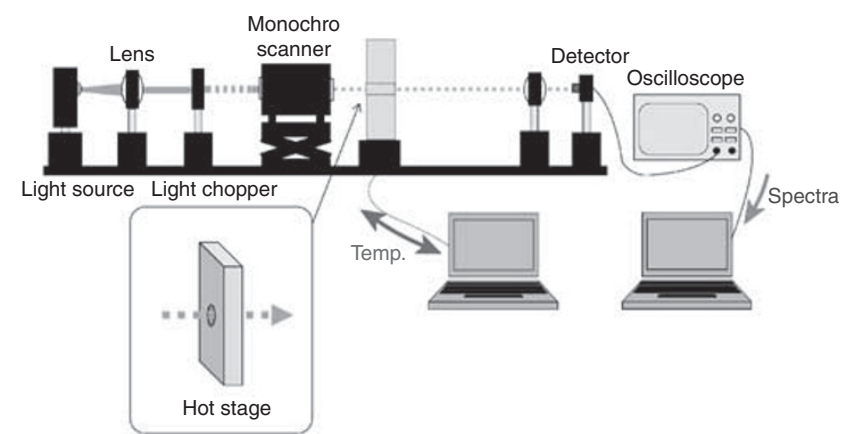

Figure 1 NIR experimental setup. A full color version of this figure is available at Polymer Journal online. oscilloscope and monochromator were analyzed with a program written using the Igor 4.0 software package (Wavematrix Inc., Portland, OR, USA). A GP-IB board (CONTEC Co. Ltd., Osaka, Japan, GP-IB(PCI)F) was used for communication between the oscilloscope, monochromator and computer.

A hot stage (Mettler Toredo International Inc., Greifensee, Switzerland) was placed in the optical pathway. NIR spectra were monitored during heating from $313-473 \mathrm{~K}$ at a rate of $2 \mathrm{~K} \mathrm{~min}^{-1}$. Spectra were measured at a sweep rate of $600 \mathrm{~nm} \mathrm{~min}^{-1}$ over the range $1650-1900 \mathrm{~nm}$, where the absorption of the first $\mathrm{CH}$ stretching overtone vibration can be detected. ${ }^{16}$ Many overlapped absorption bands make it difficult to assign the absorption bands of NIR spectra. For the assignment of NIR bands of PE, we compared NIR spectra of various linear alkane solvents from hexane to tetradecane to that of molten linear HDPE. In addition, we computed the spectra of the fundamental molecular vibration of a linear C14 hydrocarbon and a C14 hydrocarbon with an ethyl group branch at the center of the main chain by using the Gaussian $03 \mathrm{~W}$ Version 6.0 (GAUSSIAN Inc., Wallingford, CT, USA) software package. On the basis of the above measurements and computation in addition to published data, ${ }^{16,17,19}$ we determined the peak assignments of the major NIR bands for PE.

\section{RESULTS AND DISCUSSION}

NIR spectra for PE samples in the 1650-1900 nm range are shown in Figure 2; there are two predominant peaks at 1728 and $1764 \mathrm{~nm}$ and various shoulders around these peaks. The intensity of the $1728 \mathrm{~nm}$ peak for HDPE with higher crystallinity is larger than the intensity of this peak for LLDPE and LDPE, whereas the $1764 \mathrm{~nm}$ peak intensity is comparable among the three PE samples. The intensity of the $1750 \mathrm{~nm}$ peak of LDPE and LLDPE is larger than that of HDPE. Peaks at 1728 and $1764 \mathrm{~nm}$ for LDPE and LLDPE are broadened in comparison to those for HDPE, which led us to conclude that the two predominant peaks include shoulder peaks associated with chain branching. According to our previous estimation of $\mathrm{CH}_{3}$-stretching IR absorptions, the first $\mathrm{CH}_{3}$ stretching overtone vibration occurs in this region. ${ }^{21}$

To assign NIR bands to PE, we measured NIR spectra of various linear alkane solvents from hexane to tetradecane, along with molten HDPE. These spectra were measured using optical path lengths of 2 and $0.65 \mathrm{~mm}$, respectively. The intensity was divided by the thickness to yield thickness-independent results. The spectra of various alkanes and molten HDPE are shown in Figure 3.

The absorbance of the 1728 and $1764 \mathrm{~nm}$ peaks increases with increasing carbon atoms in the main chain, which suggests these peaks arise from the first $\mathrm{CH}_{2}$ stretching overtone vibration of the main chain. Figure 3 shows that the predominant peaks at 1728 and $1764 \mathrm{~nm}$ broaden with decreasing carbon atoms in the main chain. The $1750 \mathrm{~nm}$ absorption peak of the solvents is more intense than that for molten HDPE, and the valley between the 1728 and $1764 \mathrm{~nm}$ peaks deepens as the number of carbon atoms increases. This finding suggests that some absorptions around the $1740-1750 \mathrm{~nm}$ valley result

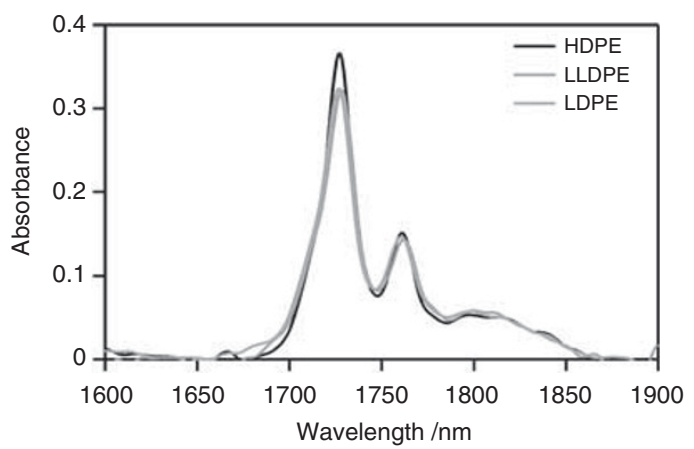

Figure 2 NIR spectra of PE samples. A full color version of this figure is available at Polymer Journal online. 


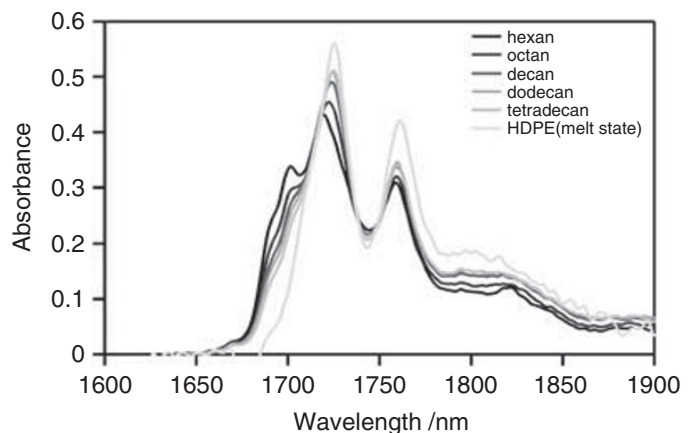

Figure 3 NIR spectra of alkane solvents and molten HDPE of varying lengths. A full color version of this figure is available at Polymer Journal online.

from $\mathrm{CH}_{3}$ vibration. Shoulder peaks at 1690 and $1710 \mathrm{~nm}$ are apparent for linear alkanes but are largely absent in the spectrum of HDPE. Ozaki et al. reported that the $1710 \mathrm{~nm}$ absorption is associated with $\mathrm{CH}_{2}$ units in the amorphous phase. ${ }^{17,19}$ It is likely that the 1690 and $1710 \mathrm{~nm}$ absorptions are associated with the $\mathrm{CH}_{3}$ groups of chain ends because the intensity of these absorptions decreases with increasing carbon atoms.

The fundamental molecular vibrations of methylene chains corresponding to each wavelength peak were calculated using the Gaussian 03W Version 6.0 (GAUSSIAN) software package, to confirm the assignment of these NIR bands. IR spectra calculated by the Gaussian software were converted to the vibrational overtone spectra, by adjusting them to the experimental NIR spectrum of the HDPE melt. Calculated spectra for a linear C14 hydrocarbon and C14 hydrocarbon with a central ethyl group branch were obtained. These spectra showed that the 1728 and $1764 \mathrm{~nm}$ peaks correspond to the first overtone of the $\mathrm{CH}_{2}$ asymmetric and symmetric stretching vibrations of the main chain, respectively. This result is consistent with assignments in previous reports. ${ }^{16}$ The calculated spectra indicate that the $1764 \mathrm{~nm}$ peak becomes more intense in the trans state and that absorption at $1750 \mathrm{~nm}$ arises from the symmetric vibration of $\mathrm{CH}_{3}$ and gauche conformation $\mathrm{CH}_{2}$ units of the main chain. The simulation results also indicate that the $\mathrm{CH}_{3}$ vibration occurs in the 1690-1710 nm region. The $1690 \mathrm{~nm}$ peak was assigned to the $\mathrm{CH}_{3}$ asymmetric vibration of the short-chain branches, and the peak at $1710 \mathrm{~nm}$ was assigned to the symmetric vibration of terminal $\mathrm{CH}_{3}$ groups. Computational assignments of NIR peaks for linear C14 and ethyl-branched C14 molecules are summarized in Table 3. These assignments support our earlier conclusions from experimental results that shoulder peaks at 1690,1710 and $1750 \mathrm{~nm}$ are largely absent in molten HDPE, which has few terminal $\mathrm{CH}_{3}$ groups. Figure 3 shows that the NIR bands at 1710 and $1690 \mathrm{~nm}$ associated with the $\mathrm{CH}_{3}$ groups of LLDPE and LDPE shift to 1715 and $1705 \mathrm{~nm}$, respectively.

Figure 4 shows NIR spectra under a constant heating rate. The intensity of the $1728 \mathrm{~nm}$ peak decreases, and that of the $1764 \mathrm{~nm}$ peak increases with increasing temperature. The magnitude of the intensity change depends on the weight fraction of the crystalline phase before melting. The temperature sensitivity of the 1728 and $1764 \mathrm{~nm}$ peaks of HDPE is considerably higher than those of LDPE and LLDPE.

The 1650-1900 nm NIR region is thought to be composed of 5 peaks at 1705, 1715, 1728, 1750 and $1764 \mathrm{~nm}$. Spectral decomposition was performed using the Voigt function implemented in the Igor 4.0 software package (Wavematrix). Figure 5 shows the component peaks obtained from curve fitting. The intensities of the 1705, 1715 and $1750 \mathrm{~nm}$ peaks of LDPE and LLDPE are greater than those of HDPE. This is due to the high terminal $\mathrm{CH}_{3}$ group content of LDPE and
Table 3 Assignments of near-infrared absorbance peaks of $\mathrm{C}_{14} \mathrm{H}_{30}$ and 7-ethyl- $\mathrm{C}_{14} \mathrm{H}_{29}$

\begin{tabular}{ll}
\hline Wavelength $(\mathrm{nm})$ & Assignments \\
\hline 1764 & $\mathrm{C}-\mathrm{H}$ stretching first overtone $\left(\mathrm{CH}_{2}\right) \mathrm{t}$ \\
1750 & $\mathrm{C}-\mathrm{H}$ stretching first overtone $\left(\mathrm{CH}_{3}\right)$ terminal $+\left(\mathrm{CH}_{2}(\mathrm{~g})\right)$ \\
1728 & $\mathrm{C}-\mathrm{H}$ stretching first overtone $\left(\mathrm{CH}_{2}\right)$ \\
1710 & $\mathrm{C}-\mathrm{H}$ stretching first overtone $\left(\mathrm{CH}_{3}\right)$ terminal \\
1690 & $\mathrm{C}-\mathrm{H}$ stretching first overtone $\left(\mathrm{CH}_{3}\right)$ short branch \\
\hline
\end{tabular}

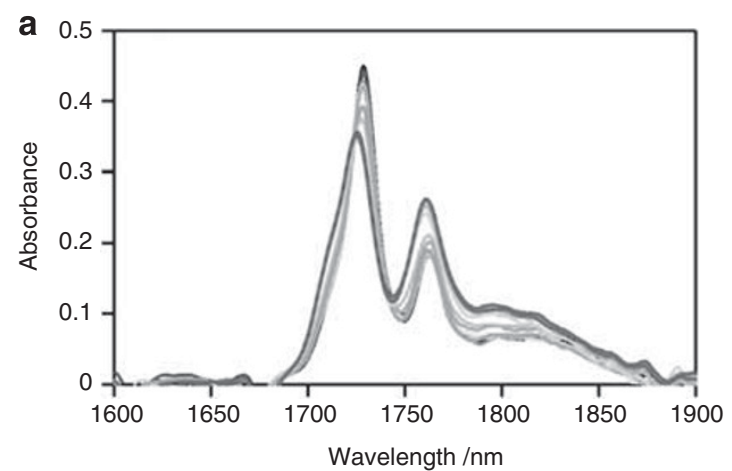

$40^{\circ} \mathrm{C}$
$200^{\circ} \mathrm{C}$

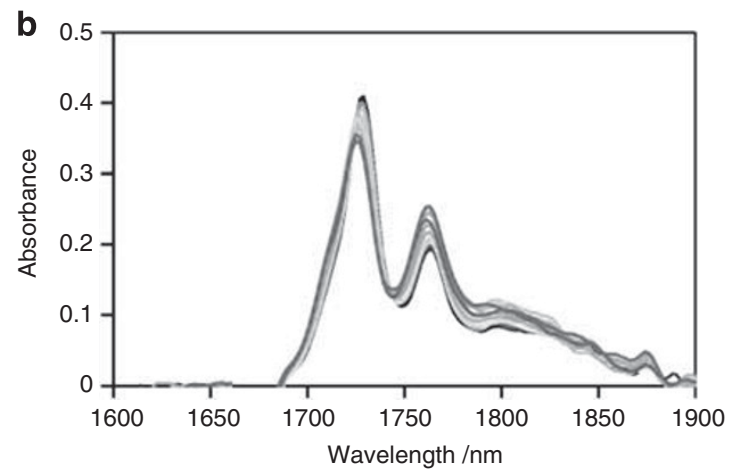

$40^{\circ} \mathrm{C}$
$200^{\circ} \mathrm{C}$

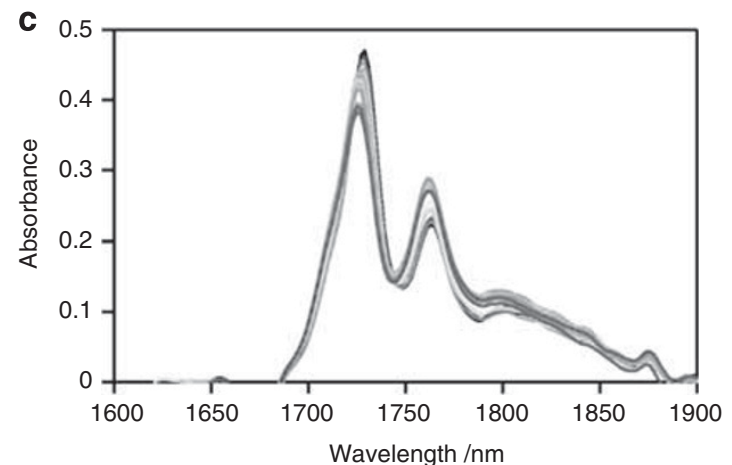

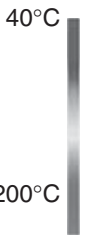

Figure 4 The temperature dependence of the NIR spectra of (a) HDPE, (b) LLDPE and (c) LDPE at a $2 \mathrm{~K} \mathrm{~min}^{-1}$ constant heating rate. A full color version of this figure is available at Polymer Journal online.

LLDPE because chain branching in this sample is much larger than that in HDPE. Figure 6 shows integrated intensities of the 1705, 1715, 1728, 1750 and $1764 \mathrm{~nm}$ peaks plotted against temperature. Each peak intensity was normalized by the total intensity of the five peaks. The DSC profile is also shown.

Figure $6 \mathrm{a}$ shows that as the temperature increases, the intensity of the HDPE CH stretching peak at $1728 \mathrm{~nm}$ decreases near the melting 

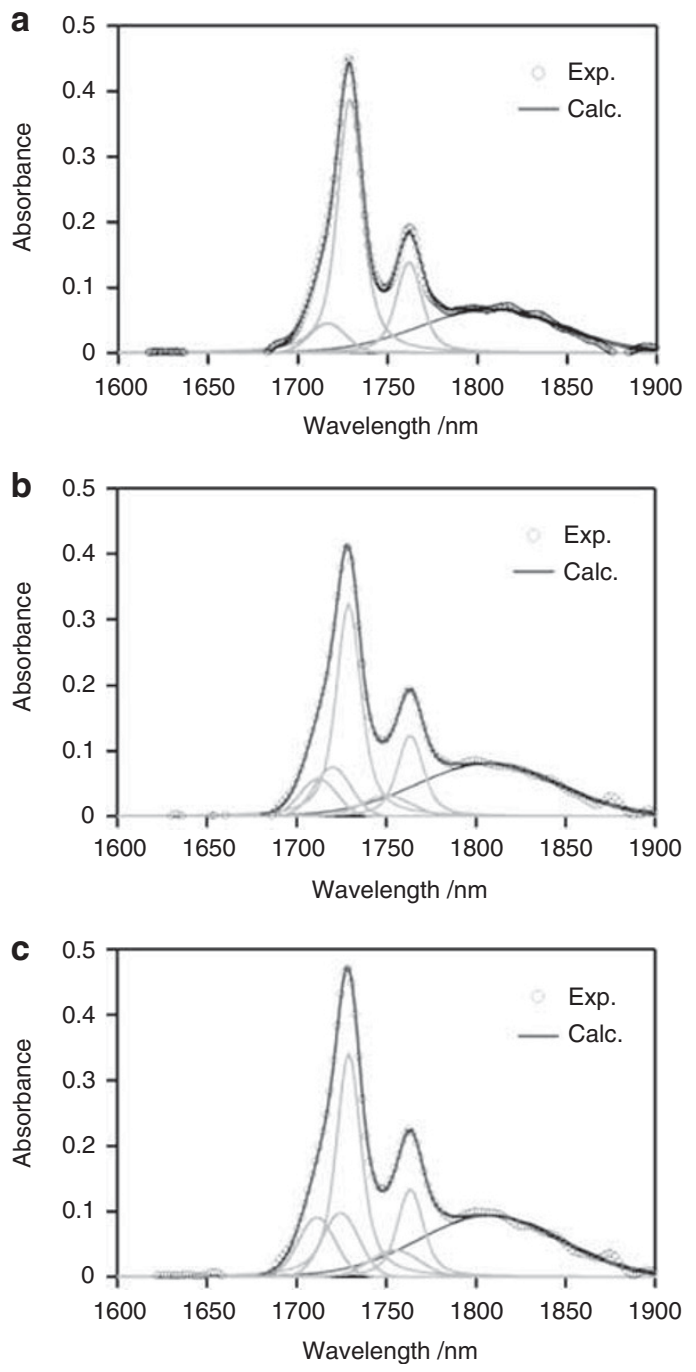

Figure 5 Typical curve-fitting results for the NIR spectra of (a) HDPE, (b) LLDPE and (c) LDPE: $\bigcirc$ denote experimental values; red lines denote the calculated spectra; orange and gray lines denote the six component peaks. A full color version of this figure is available at Polymer Journal online.

point of the DSC curve. This suggests that the asymmetric $\mathrm{CH}_{2}$ stretching vibration in the crystal lattice becomes active upon melting. In contrast, the intensity of the 1715 and $1750 \mathrm{~nm}$ peaks ascribed to terminal $\mathrm{CH}_{3}$ and gauche conformation $\mathrm{CH}_{2}$ units in the main chain, respectively, exhibits a stepwise increase near the DSC melting peak. This result suggests that melting releases the chain ends within crystalline lamellae and cilia chains embedded into crystalline lamellae, thus making it possible to change from the trans to gauche conformations. The $1705 \mathrm{~nm}$ peak ascribed to the $\mathrm{CH}_{3}$ group of short branches is too weak to detect for HDPE.

The intensity of the LLDPE peak at $1728 \mathrm{~nm}$ continuously decreases, reflecting the broad melting behavior seen in the DSC profiles in Figure $6 \mathrm{~b}$. In contrast to HDPE, the intensity of the LLDPE terminal $\mathrm{CH}_{3}$ peak at the $1715 \mathrm{~nm}$ peak is largely unchanged near the melting point, indicating that the molecular mobility of chain ends in the solid state is similar to that in the melted state. It suggests that there are many dangling chains or long cilia chains within the amorphous layer, which is plausible because the crystallinity of LLDPE is much lower than that of HDPE. The $1705 \mathrm{~nm}$ peak intensity
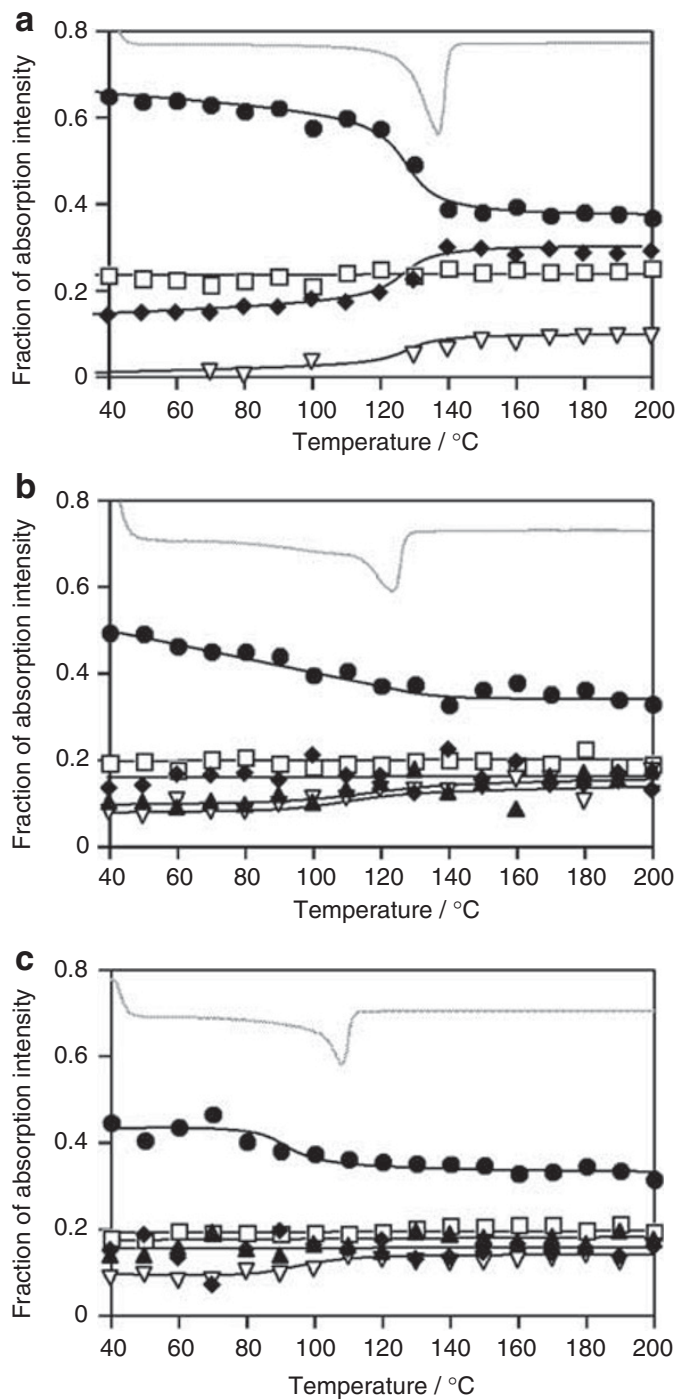

Figure 6 Variation of the fraction of NIR peak intensity for (a) HDPE, (b) LLDPE and (c) LDPE under a $2 \mathrm{~K} \mathrm{~min}^{-1}$ constant heating rate and DSC curves: the symbols denote the fraction of NIR intensity $(\mathbf{\Lambda}: 1690 \mathrm{~nm}$, : $1710 \mathrm{~nm}, \bullet: 1728 \mathrm{~nm}, \nabla: 1750 \mathrm{~nm}, \square: 1764 \mathrm{~nm}$ ); the gray line denotes the DSC curve.

(short branches) slightly increases near the DSC melting peak, indicating the inclusion of short-chain branches in the crystal lattice before melting. ${ }^{2}$

Figure $6 \mathrm{c}$ shows that the $1705 \mathrm{~nm}$ peak intensity is almost independent of melting, and that the $1728 \mathrm{~nm}$ peak intensity decreases near the DSC melting peak for LDPE. This behavior is similar to that for HDPE but different from that of LLDPE, indicating that most branches of LDPE are sufficiently long that chain ends and junctions are completely excluded from the crystal lattice and exist in the thick amorphous layers. The monotonous decrease of the $1728 \mathrm{~nm}$ peak for LLDPE suggests the inclusion of defects or comonomers in the crystalline lattice.

The $1764 \mathrm{~nm}$ peak intensity $\left(\mathrm{CH}_{2}\right.$ trans) in HDPE, LLDPE and LDPE is independent of temperature, suggesting that it is not sensitive to the melting of the crystal lattice. It appears that a certain concentration of trans conformation sequences exists that are independent of heating, up to the melting point. 


\section{CONCLUSIONS}

The NIR spectra of PE materials during heating were acquired to directly examine the molecular behavior during melting. The utility of NIR spectroscopy in investigating conformational changes of different crystallizable PE samples has been demonstrated. NIR bands of PE in the $1650-1900 \mathrm{~nm}$ region were assigned with the aid of computersimulated molecular vibrations and the experimentally measured NIR spectra of alkane solvents. The following conclusions were reached:

(1) Peaks at 1728 and $1764 \mathrm{~nm}$ result from the first overtone of the $\mathrm{CH}_{2}$ asymmetric and symmetric stretching vibrations of the main chain, respectively. The $1764 \mathrm{~nm}$ peak has a high spectral intensity in the trans state, and absorption at $1750 \mathrm{~nm}$ is associated with the symmetric vibration of $\mathrm{CH}_{3}$ and gauche conformation of $\mathrm{CH}_{2}$ groups of the main chain. The $1705 \mathrm{~nm}$ peak was assigned to the $\mathrm{CH}_{3}$ asymmetric vibration of short branches, and that at $1715 \mathrm{~nm}$ was assigned to the terminal $\mathrm{CH}_{3}$ symmetric vibration.

(2) The $1728 \mathrm{~nm}$ peak is associated with $\mathrm{CH}_{2}$ units within the crystal lattice. The molecular motion of HDPE chain ends becomes active near the melting point. The chain ends and junctions of LDPE are completely excluded from the crystal lattice and exist in the thick amorphous layer. In contrast, the crystal lattice of LLDPE includes defects or comonomers. The $1764 \mathrm{~nm}$ peak intensity is resistant to heating and melting, suggesting that a constant amount of trans sequence $\mathrm{CH}_{2}$ units always exists.

1 Zhu, X., Yan, D. \& Fang, Y. In Situ FTIR spectroscopic study of the conformational change of isotactic polypropylene during the crystallization process. J. Phys. Chem. B 105, 12461-12463 (2001).

2 Plass, M., Streck, R., Nieto, J. \& Siesler, H. W. Rheo-optical FT-IR spectroscopy of LLDPE: effect of comonomer and composite materials. Macromol. Symp. 265, 166-177 (2008).

3 Parthasarthy, G., Sevegney, M. \& Kannan, R. M. Rheooptical Fourier transform infrared spectroscopy of the deformation behavior in quenched and slow-cooled isotactic polypropylene films. J. Polym. Sci. B 40, 2539-2551 (2002).

4 Ben-Gera, I. \& Norris, K. H. Direct spectrophotometric determination of fat and moisture in meat products. J. Food Sci. 33, 64-67 (1968).
5 Barton II, F. E., Himmelsbach, D. S., Duckwoeth, J. H. \& Smith, M. J. Two-dimensional vibration spectroscopy: correlation of mid- and near-infrared regions. Appl. Spectrosc. 46, 420-429 (1992).

6 Noda, I., Dowrey, A. E. \& Marcott, C. Recent developments in two-dimensional infrared (2D IR) correlation spectroscopy. Appl. Spectrosc. 47, 1317-1323 (1993).

7 Noda, I., Liu, Y., Ozaki, Y. \& Czarnecki, M. A. Two-dimensional Fourier transform nearinfrared correlation spectroscopy studies of temperature-dependent spectral variations of Oleyl alcohol. J. Phys. Chem. 99, 3068-3073 (1995).

8 Noda, I. Determination of two-dimensional correlation spectra using the Hilbert transform. Appl. Spectrosc. 54, 994-999 (2000).

9 Hoffmannn, U., Pfeifer, F., Okretic, S., Völky, N., Zahedi, M. \& Siesler, H. W. Rheooptical Fourier transform infrared and Raman spectroscopy of polymers. Appl. Spectrosc. 47, 1531-1539 (1993).

10 Schade, C., Heckmann, W., Borchert, S. \& Siesler, H. W. Determination of orientational states in impact-polystyrene specimens by near-infrared polarization spectroscopy. Polym. Eng. Sci. 46, 381-383 (2006).

11 Watari, M. \& Ozaki, Y. Prediction of ethylene content in melt-state random and block polypropylene by near-infrared spectroscopy and chemometrics: comparison of a new calibration transfer method with a slope/bias correction method. Appl. Spectrosc. 58, 1210-1218 (2004).

12 Watari, M. \& Ozaki, Y. Calibration models for the vinyl acetate concentration in ethylene-vinyl acetate copolymers and its on-line monitoring by near-infrared spectroscopy and chemometrics: use of band shifts associated with variations in the vinyl acetate concentration to improve the models. Appl. Spectrosc. 59, 912-919 (2005).

13 Watari, M. \& Ozaki, Y. Practical calibration correction method for the maintenance of an on-line near-infrared monitoring system for molten polymers. Appl. Spectrosc. 60, 529-538 (2006).

14 Miller, C. E. Near-infrared spectroscopy of synthetic polymers. Appl. Spectrosc. Rev. 26, 277-339 (1991)

15 Furukawa, T., Watari, M., Siesler, H. W. \& Ozaki, Y. Discrimination of various poly(propylene) copolymers and prediction of their ethylene content by near-infrared and Raman spectroscopy in combination with chemometric methods. J. Appl. Polym. Sci. 87, 616-625 (2003).

16 Sato, H., Shinoyama, M., Kamiya, T., Amari, T., Šašić, S., Ninomiya, T., Siesler, H. W. \& Ozaki, Y. Near infrared spectra of pellets and thin films of high-density, low-density and linear low-density polyethylenes and prediction of their physical properties by multivariate data analysis. J. Near Infrared Spectrosc. 11, 309-321 (2003).

17 Watanabe, S., Dybal, J., Tashiro, K. \& Ozaki, Y. A near-infrared study of thermally induced structural changes in polyethylene crystal. Polymer 47, 2010-2017 (2006).

18 Watanabe, S., Noda, I. \& Ozaki, Y. Thermally induced conformational changes in polyethylene studied by two-dimensional near-infrared-infrared hetero-spectral correlation spectroscopy. J. Mol. Struct. 883-884, 173-180 (2008).

19 Watanabe, S., Noda, I. \& Ozaki, Y. Thermally induced conformational and structural disordering in polyethylene crystal studied by near-infrared spectroscopy. Polymer $\mathbf{4 9}$, 774-784 (2008).

20 Wunderlich, B. \& Dole, M. Specific heat of synthetic high polymers. VIII. Low pressure polyethylene. J. Polym. Sci. 24, 201-213 (1957).

$21 \mathrm{Krimm}, \mathrm{S}$. Infrared spectra of high polymers. Fortschr. Hochpolym. Forch. 2, 51 (1960).

22 Salazer, J. M. \& Calleja, F. J. B. On the Inclusion of Chain Defects in the Polyethylene Lattice A statistical Approach. Polym. Bull. 2, 163-167 (1980). 